\title{
Opioid Policy and Chronic Pain Treatment Access Experiences: A Multi-Stakeholder Qualitative Analysis and Conceptual Model
}

\author{
Stephanie Slat $\mathbb{D}^{\prime}$ \\ Avani Yaganti ${ }^{1}$ \\ Jennifer Thomas' \\ Danielle Helminski' \\ Michele Heisler ${ }^{1-4}$ \\ Amy Bohnert $2,4,5$ \\ Pooja Lagisetty ${ }^{1,2,4}$ \\ 'Department of Internal Medicine, \\ University of Michigan, Ann Arbor, MI, \\ USA; ${ }^{2}$ Center for Clinical Management \\ and Research, Ann Arbor VA Hospital, \\ Ann Arbor, MI, USA; ${ }^{3}$ Department of \\ Health Behavior \& Health Education, \\ University of Michigan School of Public \\ Health, Ann Arbor, MI, USA; ${ }^{4}$ Institute \\ for Healthcare Policy and Innovation, \\ University of Michigan, Ann Arbor, MI, \\ USA; ${ }^{5}$ Departments of Anesthesiology \\ and Psychiatry, University of Michigan, \\ Ann Arbor, MI, USA
}

Correspondence: Stephanie Slat

Department of Internal Medicine, University of Michigan, NCRC BI6-Floor 4, 2800

Plymouth Road, Ann Arbor, MI, 48I09, USA

Tel +l 734-755-1574

Email stslat@med.umich.edu
Purpose: Patients on long-term opioid therapy (LTOT) for pain have difficulty accessing primary care clinicians who are willing to prescribe opioids or provide multimodal pain treatment. Recent treatment guidelines and statewide policies aimed at reducing inappropriate prescribing may exacerbate these access issues, but further research is needed on this issue. This study aimed to understand barriers to primary care access and multimodal treatment for chronic pain from the perspective of multiple stakeholders.

Methods: Qualitative, semi-structured phone interviews were conducted with adult patients with chronic pain, primary care clinicians, and clinic office staff in Michigan. Interview questions covered stakeholder experiences with prescription opioids, opioid-related policies, and access to care for chronic pain. Interviews were coded using inductive and deductive methods for thematic analysis.

Results: A total of 25 interviews were conducted (15 patients, 7 primary care clinicians, and 3 office staff). Barriers to treatment access were attributed to six themes: (1) reduced clinic willingness to manage prescribed opioids for new patients; (2) lack of time and reimbursement for quality opioid-related care; (3) paucity of multimodal care and coordination between providers; (4) fear of liability and use of new guidelines to justify not prescribing opioids; (5) delayed prescription receipt due to prior authorization and pharmacy issues; and (6) poor availability of effective non-opioid treatments.

Conclusion: Issues of policy, logistics, and clinic-level resources converge to disrupt treatment access for patients with chronic pain, as many clinics both do not offer multimodal pain care and are unwilling to prescribe LTOT. The resulting conceptual model can inform the development of policy interventions to help mitigate these access barriers.

Keywords: opioid, chronic pain, access, primary care, policy

\section{Introduction}

Approximately 50 million Americans experience chronic pain. ${ }^{1}$ Many receive longterm opioid therapy (LTOT) for pain management ${ }^{2}$ in primary care settings. ${ }^{3}$ Primary care is a crucial engagement point in multimodal chronic pain treatment, the standard of care recommended by a 2019 federal task force. ${ }^{4}$ However, patients on LTOT for chronic pain experience barriers to finding new primary care clinicians, continuing existing opioid therapy with new clinicians, ${ }^{5,6}$ and accessing multimodal pain treatment. ${ }^{7}$

Multi-level factors may contribute to these access barriers. ${ }^{8}$ Low clinician confidence in treating chronic pain may make it difficult for patients to find a 
provider. ${ }^{9}$ Additionally, patients with chronic pain commonly experience stigma, manifesting as clinician disbelief in their pain, ${ }^{10,11}$ a view that opioids are illegitimate treatment, ${ }^{12}$ or assumption of an opioid use disorder. ${ }^{13-15}$ This stigma may affect clinician-patient relationships and further reduce the number of clinicians willing to treat chronic pain. Stigma also can influence governmental policies, guidelines, and health-system factors, limiting access to effective care. ${ }^{8}$ Recent guidelines aimed at reducing inappropriate opioid prescribing, such as the 2016 guidelines from the Centers for Disease Control and Prevention, ${ }^{16}$ may be perceived as creating primary care access barriers.

Research on the impacts of new opioid prescribing policies largely has reported patient and primary care clinician perspectives independently. ${ }^{13,17,18}$ Uncertainty around these policies strains the patient-provider relationship, ${ }^{19-21}$ but knowledge about policy impacts on primary care access and multimodal pain treatment access in general is limited. ${ }^{5}$ To address this gap, we evaluated how changes in policies have impacted care access, defined as the ability to be seen and treated by a clinician for pain-related needs, including long-term opioid therapy. We sought to understand the impacts of this opioid policy environment from perspectives of three key stakeholders: patients, primary care clinicians, and clinic office staff. We included office staff as they are often the first point of contact between a patient and the clinic directly with patients while scheduling appointments, requesting refills, and facilitating referrals. ${ }^{5}$ Using these findings, we created a conceptual model illustrating how specific structural issues contribute to breakdowns in access for this patient population.

\section{Methods}

Semi-structured interviews were conducted May-October 2019 with patients with chronic pain, primary care clinicians, and office staff. The University of Michigan Institutional Review Board approved this study under federal exemption 2, which applies to research which collects data only through qualitative interviews. ${ }^{22}$

\section{Recruitment}

Clinicians (physicians, physician assistants, and nurse practitioners able to prescribe opioids) and office staff were recruited by calling 189 Michigan primary care clinics from a frequently updated healthcare database. ${ }^{23}$ Each clinic was audited in a previous study ${ }^{5}$ to assess if they were willing to see a new patient requesting opioids for chronic pain, and if they were accepting patients with private insurance and Medicaid.

Research assistants (RAs) called each clinic, explained study goals, and asked to interview a clinician or staff member responsible for scheduling appointments. If available, the interview was completed immediately. Otherwise, the interview was scheduled at a later time. If the clinic requested additional information, the RA faxed a one-page flyer containing the study summary, participant incentive information, and researchers' contact information.

Patients were recruited in two ways. First, the study team placed an advertisement on an institutional health research recruiting site, which links community participants to health research studies. ${ }^{24}$ On this site, interested participants create profiles to share relevant information with study teams, including demographics and medical history. The advertisement included study goals, eligibility criteria, screening questions, and the study team's contact information. Second, the study team posted a one-page flyer throughout high-traffic areas of a large academic medical center August-September 2019. The flyer included a brief study description, eligibility criteria, and incentive information. To be eligible for the study, patients had to be adult Michigan residents, have self-reported chronic pain, and have experienced difficulty receiving opioid medication. We amended our inclusion criteria for patients towards the end of our sampling window to exclusively recruit men, with the goal of balancing the gender makeup of our sample.

\section{Interviews}

The principal investigator (PI) and RAs developed 30minute qualitative interview guides for each stakeholder type (Appendix). Questions were informed by clinician experience, text of opioid-related policies and guidelines, and open-ended responses from previous clinic audits. ${ }^{5,25}$ Interview topics included experiences with prescribing and receiving opioids, perceptions of opioid-related policies, and access to non-opioid and multimodal treatment. Participant characteristics were collected through selfreport. For clinician and staff participants, clinic ZIP code was obtained through the clinic database to determine rural or urban practice setting, as defined by the US Census. $^{26}$

After the lead RA conducted five pilot interviews, the team modified the guides, and three RAs were trained to conduct interviews. We aimed to conduct interviews until 
we felt that thematic saturation was achieved and new interviews ceased to elicit novel themes.

The University of Michigan IRB approved all aspects of our recruitment study protocol, including verbal consenting of subjects for phone interviews. All participants were informed of the purposes of this study. Patient participants were emailed IRB-approved consent forms ${ }^{27}$ and verbally consented to participate prior to the telephone interviews. As office staff and clinicians were recruited via telephone, they were read the consent form and verbally consented to participate in this study and to the publishing of anonymized results. All patient participants were sent follow-up emails containing resources regarding safe opioid use and substance use disorder treatment. Each subject received a $\$ 50$ gift card.

\section{Thematic Analysis}

Two recordings of each interview were taken to ensure adequate quality. Recordings were professionally transcribed, and transcripts were uploaded to Dedoose software (Dedoose Version 8.2.14 Los Angeles, CA: SocioCultural Research Consultants, LLC) for content analysis. Descriptive statistics were produced to describe the general characteristics of the primary care clinics as well as participant demographics. Inductive and deductive methods were used to develop a set of codebooks from preliminary transcript review and the overall research goals. ${ }^{28-30}$ Two RAs (SS, AY) independently coded each interview transcript. Under PI guidance, RAs met regularly to review coding and amend definitions. Once consensus was reached, the RAs and PI worked to develop a set of emergent themes informed by the data and prior literature. These themes and prior knowledge were then used to develop a conceptual model of treatment access for this patient population. The chronological order in which many patients receive care was identified, and the emergent themes were then mapped to the corresponding parts of the care process. The model was further developed through a process of iterative feedback from members of the research team and other experts on this subject area.

\section{Results}

\section{Participant Characteristics}

Twenty-five interviews were conducted with 15 patients, 7 clinicians, and 3 office staff (Table 1). Median interview length was 20 minutes, with interviews ranging from 11 to 52 minutes. The study received 29 interested patient
Table I Characteristics of Interview Respondents

\begin{tabular}{|c|c|}
\hline & N (Percentage) \\
\hline Patients & 15 (100\%) \\
\hline \multicolumn{2}{|l|}{ Sex } \\
\hline Male & $4(26.7 \%)$ \\
\hline Female & II (73.3\%) \\
\hline \multicolumn{2}{|l|}{ Age } \\
\hline $35-45$ & $5(33.3 \%)$ \\
\hline $46-55$ & 5 (33.3\%) \\
\hline $56-65$ & $4(26.7 \%)$ \\
\hline$\geq 66$ & I (6.7\%) \\
\hline \multicolumn{2}{|l|}{ Race } \\
\hline White & $10(66.7 \%)$ \\
\hline Black & $4(26.7 \%)$ \\
\hline Other/Multiple races & I (6.7\%) \\
\hline \multicolumn{2}{|l|}{ Education level } \\
\hline High school diploma or GED & 7 (46.7\%) \\
\hline Associate's degree & $0(0.0 \%)$ \\
\hline Bachelor's degree & $4(26.7 \%)$ \\
\hline Graduate or professional degree & $4(26.7 \%)$ \\
\hline \multicolumn{2}{|l|}{ Setting } \\
\hline Rural & $6(40 \%)$ \\
\hline Urban & $9(60 \%)$ \\
\hline Clinicians & 7 (100\%) \\
\hline \multicolumn{2}{|l|}{ Sex } \\
\hline Male & 5 (7I.4\%) \\
\hline Female & $2(28.6 \%)$ \\
\hline \multicolumn{2}{|l|}{ Role } \\
\hline Physician & $4(57.1 \%)$ \\
\hline Nurse practitioner & $2(28.6 \%)$ \\
\hline Physician's assistant & I (I4.3\%) \\
\hline \multicolumn{2}{|l|}{ Practice Setting } \\
\hline Rural & $4(57.1 \%)$ \\
\hline Urban & $3(42.9 \%)$ \\
\hline Office Staff & $3(100 \%)$ \\
\hline \multicolumn{2}{|l|}{ Sex } \\
\hline Male & $0(0.0 \%)$ \\
\hline Female & $3(100.0 \%)$ \\
\hline \multicolumn{2}{|l|}{ Role } \\
\hline Office Manager & $2(66.7 \%)$ \\
\hline Scheduler & I (33.3\%) \\
\hline \multicolumn{2}{|l|}{ Practice Setting } \\
\hline Rural & I (33.3\%) \\
\hline Urban & $2(66.7 \%)$ \\
\hline
\end{tabular}

participants, and 15 were included. One patient participated in a pilot interview, five were deemed ineligible to participate, and eight did not respond after 3 contact 
attempts from the study team. Of interviewed patients, 14 had been recruited through the recruitment site and 1 had been recruited via flyer. Most were female $(n=11)$ and white $(\mathrm{n}=10)$. About half of patients $(\mathrm{n}=8)$ held a bachelor's or graduate degree; the remaining 7 held a high school diploma as their highest degree. Six patients lived in rural regions while 9 lived in urban regions. The median patient age was 49 , with a minimum age of 35 and a maximum of 69 .

Three office staff and 7 primary care clinicians were interviewed, representing 7 different clinics. Four clinics were in urban regions. Five clinics were considered small ( 2 or fewer clinicians on site) and two large ( 3 or more clinicians). When audited in a prior study, ${ }^{5}$ two of 7 clinics indicated unwillingness to prescribe opioids for new patients currently taking opioids for pain, and five said they would be willing to continue prescribing. Five of the 7 primary care clinicians interviewed were male. Clinicians included 4 physicians, 2 physician assistants, and 1 nurse practitioner. All interviewed office staff were female, with 2 identifying as office managers and 1 as a scheduler.

\section{Key Themes}

Treatment access barriers were attributed to six major themes: (1) reduced clinic willingness to manage prescribed opioids for new patients, (2) lack of time and reimbursement for quality opioid-related care, (3) paucity of multimodal care and coordination between providers, (4) fear of liability and use of new guidelines to justify not prescribing opioids, (5) delayed prescription receipt due to prior authorization and pharmacy issues, and (6) poor availability of effective non-opioid treatments. These themes informed the development of a conceptual model of chronic pain treatment access through primary care.

\section{Reduced Clinic Willingness to Manage Prescribed Opioids for New Patients}

Clinicians and office staff made statements demonstrating their clinic's reduced willingness to manage LTOT for new patients compared to established patients. Although clinicians did not mention discharging existing LTOT patients from their practice, they often expressed reluctance to manage opioids for new patients with chronic pain. Clinicians reported frequently referring these patients directly to pain management clinics or other services.

If they have been established, then they expect to get their monthly usual prescription. If they are new, then I can insist that they go to a pain clinic or try other possibilities.

- Clinician 2

Even when clinics would manage LTOT for new patients, some implemented strict clinical requirements prior to issuing a prescription.

We have a very strict policy ... if you are new patient, we are going to send you for bloodwork ... and, it usually takes ... probably about three appointments to ... be able to get a prescription. - Office Staff 1

Patients reported barriers reflecting some of the policies clinicians and staff discussed.

Whenever I have tried to find a new doctor ... it seems like they do not even listen to your symptoms, they just block you from getting any opioids. They just let you know upfront: no opioids. - Patient 4

One respondent reported that these barriers precipitated transition to illicit opioid use.

I tried [to find a new clinician] and I was just blocked at every turn so that's what caused me to try to procure [opioids] on the street. - Patient 4

\section{Lack of Time and Reimbursement for Quality Opioid-Related Care}

Some clinicians cited insufficient reimbursement as limiting quality care access. They described a lack of time for required services, including urine drug screening and Michigan's state-mandated Opioid Start Talking form, an informed consent given prior to initiation of opioids. ${ }^{31}$

We are doing [the Michigan Automated Prescription System] ... a house drug screen ... a controlled substance agreement ... the Opioids Start Talking form ... the patient counseling guide ... it's become much more time intensive, without the compensation. - Clinician 1

So I think in the end, we send [patients requesting opioids] out to a pain management center just because they may run their clinics a little more smoothly and have everything set up with the drug screens and the, you know, protocols. - Clinician 6

This inadequate compensation and time may have also led to all or nothing decision-making, in terms of opioidrelated care. Accounts of low-quality and non-collaborative decision-making around patients' opioid treatment plans, such as sudden discontinuation, were common. 
One patient felt they were offered two treatment options: a large amount of opioids or none at all.

There were certain times that I just needed the mental break from the pain and then, I knew that I could just deal with it again ... But the answer was always you are either going to get 100 pills or you are going to get zero. It's like, well, could I just have two? - Patient 7

\section{Paucity of Multimodal Care and Coordination Between Providers}

Most clinicians and patients reflected on the complexity of chronic pain and LTOT and failures in nuanced pain care delivery, and reported a desire for better multimodal care in chronic pain treatment.

Chronic pain is a multi-system issue that requires a primary care physician, a pain specialist, and a [psychotherapist] for their mental health. We are talking about three things here. - Clinician 5

Patients also reported inadequate coordination between their primary care clinician and various specialists, creating difficulty navigating independent, and sometimes conflicting, treatment plans.

Everybody's perspective was right, but when you put it all together it was wrong ... In a whole person collaboration, it did not make any sense to me as the person. - Patient 7

In these contexts, some patients expressed feeling responsible for their own care coordination.

I am trying to focus on getting well ... and then now you are telling me that I have to figure out how to be a pharmacist for myself and I do not know how to do that, and I do not want to do that wrong. - Patient 7

\section{Fear of Liability and Use of New Guidelines to Justify Not Prescribing Opioids}

Clinic-side and patient interviews differed in reported clinician motivations for decreased opioid prescribing. Clinicians and office staff often cited fears of liability for driving changes but rarely referenced best practice guidelines or care quality concerns.

The [Drug Enforcement Administration] is kind of following things, [and there's] more liability so ... there are lots of patients that we are trying to keep off opioids ... it slows down your practice with all the paperwork you have to do." - Clinician 6.
I do not think it's the policies necessarily that have influenced these providers. I think it's just their thought process that they do not want to take these patients on because then, it [is] their licensure ... and their private practice ... if something were to happen. I think it's [the liability] that comes into play. - Office Staff 3.

Patients, however, frequently reported clinicians invoking recent guidelines as the basis for altering or discontinuing their LTOT. They gave examples of doctors referring to "state policies" or "the CDC" when justifying treatment plan changes.

The new doctor I got told me that because nothing showed up [on MRI and CAT scans], and the new laws were taking effect, and the regulation of opioid medications ... that was when the CDC claimed that there was an opioid epidemic and changed everything. - Patient 14

\section{Delayed Prescription Receipt Due to Prior Authorization and Pharmacy Issues}

Many interviewees reported logistical barriers delaying medication receipt, which can present a major barrier to timely care. Patients and clinicians encountered these logistical issues in different ways.

Clinicians primarily discussed increased administrative workload as a source of dispensing delays. Examples included completing prior authorization insurance forms and fielding calls about opioid prescriptions from pharmacists. Clinicians described pharmacists requesting more information about or challenging prescriptions to an extent beyond standards of practice, ${ }^{16}$ such as inquiring about prescription dose or reason. These clinicians felt knowledgeable about relevant guidelines and sometimes viewed these inquiries as unfounded and unnecessary.

With the pharmacy, they become more in their mind like they are prescribers, like they are allowed to question what we are doing, and why we are doing it. And in addition to ... all the documenting we have to do, and the prior [authorizations] ... we have to contend with the pharmacist trying to make our job harder. - Clinician 4

Patients reported receiving discordant explanations for prescription issues. In some cases, patients reported inconsistent explanations from the same healthcare worker regarding a medication delay. For instance, a pharmacist may say prior authorization caused a delay and later tell the patient the medication is out of stock. Other patients received different explanations from different sources, such as the pharmacist reporting a prescription writing 
error and clinicians claiming an insurance issue. These discrepancies frustrated patients as they navigated logistical hurdles and concerns about running out of medication.

The doctor's office ... kept giving me different stories as to why it was not being filled and I would keep going to the pharmacist and they would keep saying "we didn't get the prescription yet." - Patient 11

\section{Poor Availability of Effective Non-Opioid Treatments} Patients reported being offered non-opioid alternatives for chronic pain, including anti-inflammatory medications and heating pads, but frequently regarded them as unacceptable and ineffective.

They should offer alternatives instead of just saying, "Well, you can take Tylenol" ... They need to become more educated on pain management. - Patient 6

In addition, patients and clinicians described inadequate insurance coverage as a barrier to pain clinics. They reported pain management clinics not accepting Medicaid and insurance plans not covering certain procedures.

They basically just told me that, well, your "insurance doesn't cover" cervical injections and, you know, I "can't write you a script for pain medication." - Patient 14
Clinicians cited longer wait times for patients with Medicaid than for those with private insurance and reported a desire for more pain management clinics that accept Medicaid in their respective regions.

We have quite a few Medicaid patients and a lot of pain management providers do not necessarily take that, so it's a long time for them to get in. - Clinician 1

\section{A Conceptual Model of Treatment Access}

To illustrate this patient population's experiences, we propose a conceptual model of access to effective, multimodal chronic pain treatment through primary care (Figure 1). While individual pathways to effective treatment differ, we identify multiple stages where patients can encounter barriers: as they seek access to primary care, as they engage in care planning with their clinician, and as they initiate treatment. Contextual factors, including individual patient and provider attributes, health system-level factors, and governmental regulations and guidelines, can also limit access to effective treatment at any of these temporally distinct points in care. For example, in seeking primary care, reduced clinic willingness to manage prescribed opioids for new patients may pose a significant barrier. As a patient engages in care planning, both systemic barriers to high-quality pain care delivery as well as

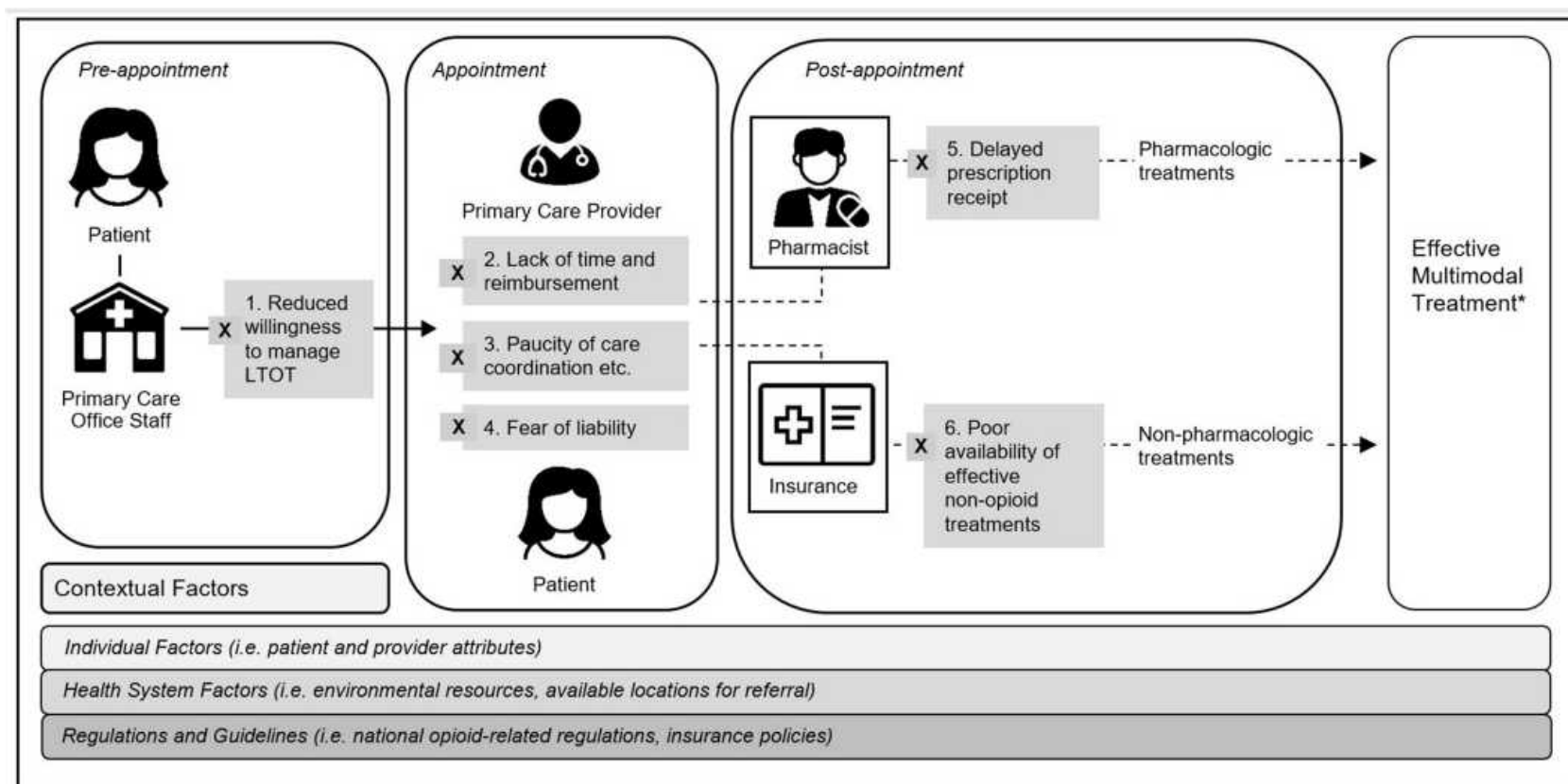

Figure I Conceptual model of treatment access for chronic pain treatment through primary care. *Effective multimodal treatment in this model refers to a multidisciplinary approach to pain management, including modalities such as medications, restorative therapies, interventional procedures, behavioral health approaches, and complementary and integrative health. 
clinician fears around opioid prescribing may limit access to effective treatment. Contextual factors such as individual-level factors on the part of both the patient and providers, as well as health system-level factors and the policy and guideline environment may affect quality care access at various points.

\section{Discussion}

In the context of recent guidelines and policies attempting to prevent opioid-related harm, ${ }^{4,16}$ this study highlights heterogeneous breakdowns in effective pain treatment access through primary care according to multiple stakeholders. While many clinical guidelines go unfollowed, ${ }^{32}$ the 2016 CDC guidelines ${ }^{16}$ were associated with substantial decreases in opioid prescribing, ${ }^{33}$ around which a fear of liability persists. Clinicians may use recent guidelines to justify not prescribing, even when prescribing may be guideline-concordant, while multiple stakeholders describe poor availability of effective non-opioid treatments. Clinicians and staff discussed reduced willingness to manage LTOT for new patients, and patients and clinicians detailed additional barriers in quality pain care delivery. When clinicians are willing to prescribe opioids for this patient population, prior authorization and pharmacy issues may delay prescription receipt.

The perception that patients on LTOT increase administrative burden and potential liability may motivate clinicians to change their opioid prescribing in ways that reduce access, including discriminating against new patients on LTOT. This finding is consistent with prior studies demonstrating patients on LTOT encounter barriers to finding a new prescriber as well as popular press reports of "opioid refugees" left without care. ${ }^{5,34}$ Ideally, patients with chronic pain would have adequate access to specialty multimodal pain care, where clinicians can focus exclusively on pain treatment. However, this care is not available to most individuals, and low Medicaid acceptance at pain clinics exacerbates access barriers. ${ }^{7}$ Although many non-pharmacologic treatments have proven to be effective, ${ }^{4}$ out-of-pocket expenses prohibit many patients from accessing these options. ${ }^{35}$ Without a clear understanding of relevant guidelines, clinicians and healthcare workers can make unfounded treatment decisions around opioids, resulting in inadequate care. Clinicians and pharmacists may also be compelled to shift blame to each other, treatment guidelines, or insurance policies to preserve relationships with patients. Our findings showed that logistical, policy, and clinic-level issues converge to restrict effective treatment access for this population.

This study addresses a gap in the literature concerning clinic perceptions of new governmental regulations around opioid prescribing for chronic pain. Including clinicians and office staff voices allows more thorough identification of key breakpoints ineffective care access. Consistent with prior studies around resource constraints, ${ }^{9}$ clinician interviewees cited a lack of time and reimbursement for caring for this patient population. Primary care visits are often scheduled to allow only 15 minutes or fewer to address multiple issues, ${ }^{36}$ and quality pain care can be timeintensive. ${ }^{4}$ In addition to resource constraints, clinicians reported an increased fear of personal liability around opioid prescribing, consistent with prior research. ${ }^{25}$ Clinicians in our study referenced fear of litigation as justification for decreasing opioid prescribing more frequently than care quality concerns, perhaps indicating a disproportionate focus on liability.

Furthermore, we found pharmacist inquiries about prescriptions can increase administrative burdens on primary care offices and contribute to opioid-related stigma. Pharmacists' inquiries may be motivated by safety concerns, yet are considered unhelpful by primary care offices. This novel finding indicates that pharmacists should be included in interventions to increase treatment access for this patient population. Improved models of physician-pharmacist care coordination show promise in enhancing communication and warrant further investigation. $^{37}$

The proposed conceptual model is informed by multiple stakeholders and synthesizes many stages of treatmentseeking into a cohesive representation. This study elucidates several path-dependent barriers patients may face as they seek care, and the emergent themes represent major breakpoints within unique domains. This model describes pain care access as a complex system, shaped by numerous participants and external factors. It highlights how many points exist during the treatment-seeking process where a patient's care access can be limited, and that many external factors, including reimbursement policies, shape the way that care is delivered. Through use of this conceptual framework, policymakers can be thoughtful about designing solutions that take these breakpoints into consideration.

Our study had several limitations. Our patient recruitment yielded a small convenience sample of mostly White female respondents, so our findings may not be 
representative of all issues patients encounter in accessing care, including racial or gender biases. It also relied on self-report of chronic pain, with no specified duration requirement. Additionally, all but one interviewed patient was recruited via an institutional health research recruitment site, which could introduce bias as our respondents are likely more experienced with health research than the general patient population. Our results are also based on a small sample of clinicians and office staff who may have outlier experiences, as we found their recruitment challenging despite iterative efforts. Despite the limited sample size, examining responses from three stakeholder groups around similar central questions elicited important perspectives. Still, as our respondents constitute a convenience sample, our results may not be generalizable to all relevant patient and provider experiences. Additionally, our research was limited to Michigan, so our findings likely do not represent experiences in other states, where policies differ.

Primary care is an important point of engagement in healthcare for patients with chronic pain. Unfortunately, many recent policies and regulations aimed at reducing mortality from the opioid epidemic have led to unintended restrictions in treatment access for this population, including access to non-pain care. ${ }^{5}$ These issues are associated with negative opioid-related outcomes such as hospitalization, suicidal ideation, and transition to illicit use. ${ }^{38-42}$ In conclusion, this study highlights heterogeneous treatment barriers, including lack of time and resources for clinicians, liability concerns, and scrutiny from pharmacists and insurers. Future interventions to address access barriers for this high-risk patient population need to address the many breakdowns in care and consider all involved stakeholders: patients, clinicians, office staff, pharmacists, and payers.

\section{Acknowledgments}

We would like to acknowledge Colin MacLeod and Adrianne Kehne for their thoughtful edits. An abstract of this paper was presented at the 42nd Annual Meeting of the Society for Medical Decision Making as a prerecorded oral presentation with interim findings. The presentation's abstract was published in the online conference catalog at https://smdm.confex.com/smdm/2020/meetin gapp.cgi/Paper/13733.

\section{Disclosure}

This work was funded by the Michigan Health Endowment Fund (PAL) and by the National Institute on Drug Abuse of the National Institutes of Health Award [grant number K23 DA047475 (PAL)]. The authors have no other conflicts of interest to report.

\section{References}

1. Dahlhamer J, Lucas J, Zelaya C, et al. Prevalence of chronic pain and high-impact chronic pain among adults - United States, 2016. MMWR Morb Mortal Wkly Rep. 2018;67(36):1001-1006. doi:10.15585/mmwr.mm6736a2

2. Boudreau D, Von Korff M, Rutter CM, et al. Trends in long-term opioid therapy for chronic non-cancer pain. Pharmacoepidemiol Drug Saf. 2009;18(12):1166-1175. doi:10.1002/pds.1833

3. Volkow ND, McLellan TA, Cotto JH, et al. Characteristics of opioid prescriptions in 2009. JAMA. 2011;305(13):1299-1301. doi:10.1001/ jama.2011.401

4. U.S. Department of Health and Human Services. Pain management best practices inter-agency task force report: updates, gaps, inconsistencies, and recommendations. U. S. Department of Health and Human Services. May, 2019. Available from: https://www.hhs.gov/ ash/advisory-committees/pain/reports/index.html. Accessed March 25, 2021.

5. Lagisetty PA, Healy N, Garpestad C, et al. Access to primary care clinics for patients with chronic pain receiving opioids. JAMA Netw Open. 2019;2(7):e196928. doi:10.1001/jamanetworkopen.2019.6928

6. Keller MS, Jusufagic A, Nuckols TK, et al. Understanding clinicians' decisions to assume prescriptions for inherited patients on long-term opioid therapy: a Qualitative Study [published online ahead of print, 2020 Mar 18]. Pain Med. 2020;pnaa045. doi:10.1093/pm/pnaa045

7. Lagisetty $\mathrm{P}$, Slat $\mathrm{S}$, Thomas $\mathrm{J}$, et al. Access to multimodal pain management for patients with chronic pain: an Audit Study. J Gen Intern Med. 2021;36(3):818-820. doi:10.1007/s11606-020-05866-3

8. Livingston JD, Boyd JE. Correlates and consequences of internalized stigma for people living with mental illness: a systematic review and meta-analysis. Soc Sci Med. 2010;71(12):2150-2161. doi:10.1016/j. socscimed.2010.09.030

9. Jamison RN, Scanlan E, Matthews ML, et al. Attitudes of primary care practitioners in managing chronic pain patients prescribed opioids for pain: a prospective longitudinal controlled trial. Pain Med. 2016;17(1):99-113. doi:10.1111/pme.12871

10. De Ruddere L, Craig K. Understanding stigma and chronic pain: a state-of-the-art review. Pain. 2016;157(8):1607-1610. doi:10.1097/j. pain.0000000000000512

11. Matthias MS, Parpart AL, Nyland KA, et al. The patient-provider relationship in chronic pain care: providers' perspective. Pain Med. 2010;11(11):1688-1697. doi:10.1111/j.1526-4637.2010.00980.x

12. McCradden MD, Vasileva D, Orchanian-Cheff A, et al. Ambiguous identities of drugs and people: a scoping review of opioid-related stigma. Int $J$ Drug Policy. 2019;74:205-215. doi:10.1016/j. drugpo.2019.10.005

13. Antoniou T, Ala-Leppilampi K, Shearer D, et al. "Like being put on an ice floe and shoved away": a qualitative study of the impacts of opioid-related policy changes on people who take opioids. Int J Drug Policy. 2019;66:15-22. doi:10.1016/j.drugpo.2019.01.015

14. Schatman ME, Shapiro H. Damaging state legislation regarding opioids: the need to scrutinize sources of inaccurate information provided to lawmakers. J Pain Res. 2019;12:3049-3053. doi:10.2147/JPR.S235366 
15. Matthias M, Krebs E, Bergman A, et al. Communicating about opioids for chronic pain. EJP. 2014;18:835-843. doi:10.1002/ j.1532-2149.2013.00426.x

16. St. Marie B. Primary care experiences of people who live with chronic pain and receive opioids to manage pain: a qualitative methodology. J Am Assoc Nurse Pract. 2016;28:429-435. doi:10.1002/ 2327-6924.12342

17. Matthias MS, Johnson NL, Shields CG, et al. "I'm not gonna pull the rug out from under you": patient-provider communication about opioid tapering. J Pain. 2017;18(11):1365-1373. doi:10.1016/j. jpain.2017.06.008

18. Gruß I, Firemark A, Mayhew M, et al. Taking opioids in times of crisis: institutional oversight, chronic pain and suffering in an integrated healthcare delivery system in the. US Int J Drug Pol Icy. 2019;74:62-68. doi:10.1016/j.drugpo.2019.08.009

19. Matthias MS, Krebs EE, Collins LA, et al. "I'm not abusing or anything": patient-physician communication about opioid treatment in chronic pain. Patient Educ Couns. 2013;93(2):197-202. doi:10.1016/j.pec.2013.06.021

20. Dowell D, Haegerich TM, Chou R. CDC guideline for prescribing opioids for chronic pain-United States, 2016. JAMA. 2016;315 (15):1624-1645. doi:10.1001/jama.2016.1464

21. Al Achkar M, Revere D, Dennis B, et al. Exploring perceptions and experiences of patients who have chronic pain as state prescription opioid policies change: a qualitative study in Indiana. BMJ Open. 2017:e015083. doi:10.1136/bmjopen-2016-015083

22. United States code of federal regulations, title 45, subtitle B, $\S 46.104$. Electronic Code of Federal Regulations. Available from: www.ecfr gov/. Accessed March 25, 2021.

23. SK\&A by IQVIA. Databases and list finder. 2019. Available from: https://www.skainfo.com/databases. Accessed March 15, 2019.

24. Doshi A, Connally L, Spiroff M, et al. Adapting the buying funnel model of consumer behavior to the design of an online health research recruitment tool. J Clin Transl Science. 2017;1(4):240 245. doi:10.1017/cts.2017.17

25. North carolina medical board licensee survey results. Available from: https://www.ncmedboard.org/images/uploads/article_images/ NCMBLicensee_Survey_July2018_-_Public.pdf. Accessed March $15,2019$.

26. Ratcliffe M, Burd C, Holder K, et al. Defining rural at the U.S. census bureau. Am Community Surv Geogr Brief. 2016;1(8). In: Bureau USC, ed.

27. IRBMED Standard Informed Consent Template. University of Michigan Medical School office of research. Available from: https://az.research.umich.edu/medschool/templates/standardinformed-consent-template. Accessed January 22, 2021.

28. Vaismoradi M, Turunen H, Bondas T. Content analysis and thematic analysis: implications for conducting a qualitative descriptive study. Nurs Health Sci. 2013;15(3):398-405. doi:10.1111/nhs.12048
29. Hsieh HF, Shannon SE. Three approaches to qualitative content analysis. Qual Health Res. 2005;15(9):1277-1288. doi:10.1177/ 1049732305276687

30. Whittemore R, Chase SK, Mandle CL. Validity in qualitative research. Qual Health Res. 2001;11(4):522-537. doi:10.1177/ 104973201129119299

31. House Bill 4408Mich Pub Act 246 (2017). Available from: http:// www.legislature.mi.gov/documents/2017-2018/publicact/htm/2017PA-0246.htm.

32. Barth JH, Misra S, Aakre KM, et al. Why are clinical practice guidelines not followed? Clin Chem Lab Med. 2016;54(7):11331139. doi:10.1515/cclm-2015-0871

33. Bohnert ASB, Guy GP, Losby JL. Opioid prescribing in the United States before and after the centers for disease control and prevention's 2016 opioid guideline. Ann Intern Med. 2018;169(6):367-375. doi:10.7326/M18-1243

34. Szalavitz M. When the cure is worse than the disease. New York Times. February 9, 2019. Available from: https:/www.nytimes.com/ 2019/02/09/opinion/sunday/pain-opioids.html. Accessed May 6, 2020.

35. Heyward J, Jones CM, Compton WM, et al. Coverage of nonpharmacologic treatments for low back pain among US public and private insurers. JAMA Netw Open. 2018;1(6):e183044. doi:10.1001/ jamanetworkopen.2018.3044

36. Wilson A, Childs S. The relationship between consultation length, process ad outcomes in general practice: a systematic review. $\mathrm{Br} J$ Gen Pract. 2002;52(485):1012-1020.

37. Lagisetty P, Smith A, Antoku D, et al. A physician-pharmacist collaborative care model to prevent opioid misuse. Am J Health Syst Pharm. 2020;77(10):771-780. doi:10.1093/ajhp/zxaa060

38. Mark TL, Parish W. Opioid medication discontinuation and risk of adverse opioid-related health care events. J Subst Abuse Treat. 2019;103:58-63. doi:10.1016/j.jsat.2019.05.001

39. Coffin PO, Rowe C, Oman N, et al. Illicit opioid use following changes in opioids prescribed for chronic non-cancer pain. PLoS One. 2020;15(5):e0232538. doi:10.1371/journal.pone.0232538

40. Martin J, Cunliffe J, Décary-Hétu D, et al. Effect of restricting the legal supply of prescription opioids on buying through online illicit marketplaces: interrupted time series analysis. BMJ. 2018;361:k2270. doi:10.1136/bmj.k2270

41. Oliva EM, Bowe T, Manhapra A, et al. Associations between stopping prescriptions for opioids, length of opioid treatment, and overdose or suicide deaths in US veterans: observational evaluation. $B M J$. 2020;368:m283. doi:10.1136/bmj.m283

42. Demidenko MI, Dobscha SK, Morasco BJ, et al. Suicidal ideation and suicidal self-directed violence following clinician-initiated prescription opioid discontinuation among long-term opioid users. Gen Hosp Psychiatry. 2017;47:29-35. doi:10.1016/j.genhosppsych.20 17.04 .011
Journal of Pain Research

\section{Publish your work in this journal}

The Journal of Pain Research is an international, peer reviewed, open access, online journal that welcomes laboratory and clinical findings in the fields of pain research and the prevention and management of pain Original research, reviews, symposium reports, hypothesis formation and commentaries are all considered for publication. The manuscript

Submit your manuscript here: https://www.dovepress.com/journal-of-pain-research-journa management system is completely online and includes a very quick and fair peer-review system, which is all easy to use. Visit http:// www.dovepress.com/testimonials.php to read real quotes from published authors. 